\title{
Bernhard Gross and the Evolution of Modern Electret Research
}

\author{
G. M. Sessler \\ Darmstadt University of Technology \\ Merckstraße 25, D-64283 Darmstadt \\ Received 3 December, 1998
}

\begin{abstract}
During his scientific career, spanning the time from the early 1930 's to the present date, Bernhard Gross has contributed to many disciplines of physics and engineering. Some of these fields, such as radiation effects in solids and charge storage and transport in dielectrics, are integral parts of electret research, an area which Gross has promoted, and whose development he has guided, over the past six decades. The present article focusses on the outstanding contributions of Bernhard Gross to electret research and discusses the impact of his work on the evolution of this field.
\end{abstract}

\section{Starting points: early electret research}

When, in the 1930's, Bernhard Gross started his early work on charge and polarization phenomena in dielectrics [1, 2], the field of electret research was still in its infancy. Earlier in the century, the Japanese physicist Eguchi had reported the first systematic experiments on electrets and had found polarity reversals and other effects [3] which puzzled scientists at that time. Another phenomenon related to electrets and much discussed in those days was dielectric absorption, i.e. the property of dielectrics to store charge when exposed to high fields [1]. These phenomena fascinated Bernhard Gross and he began, as shall be discussed below, some fundamental studies of the underlying physical phenomena. In the course of this work, he laid the groundstones to modern electret research.

During his scientific career, Bernhard Gross has contributed decisively to practically all areas of electret research and thereby stimulated the work of many other colleagues. He is equally recognized as a theoretician and as an experimentalist. As somebody who has collaborated with him on many projects, the author was always fascinated by Gross' clear grasp of scientific problems and by his ability to deal with these using appropriate theoretical descriptions or intuitive experiments. With his sustained contributions, he has guided the evolution of electret research over more than half a century and has helped to establish this field as a

respected discipline of modern science.

\section{Superposition principle and dielectric absorption}

Bernhard Gross entered the field of electrets through his work on cosmic radiation. It was well known at that time that insulator leakage, caused by the radiation, limits the sensitivity of ionization chambers. In this context, some of the early work of Gross was devoted to dielectric absorption, as already mentioned above.

Internal charging and discharging of a dielectric in an applied field is controlled by the superposition principle. This principle states that a certain variation of the external force always results in the same effect, regardless of the state of the system. On the basis of this principle, Gross was able to formulate a linear response theory for dielectrics $[1,2]$. In particular he derived fundamental relations for the currents, voltages, polarization effects, and relaxation times. These relations were instrumental for the description and understanding of dielectric absorption.

During these studies Gross also noticed that the formalisms of dielectric and viscoelastic relaxations are identical. He therefore applied the methods he had developed to viscoelasticity, discovering a new integral transform sometimes referred to as second Gross transform (the first Gross transform relates the absorption of unidirectional and isotropic beams of radiation and 
was discovered by Gross during his early work on cosmic radiation). The second transform was used extensively in the following for the description of the mechanical behavior of elastic materials [4].

\section{Two-charge theory and measuring methods}

Of great importance in the development of electret research were fundamental studies about the nature of the electret charges. Older work by Mikola [5], Adams [6] and Gemant [7] had shown that two charges of different nature and opposite polarity exist on nonmetalized dielectrics charged by voltage application to electrodes adjacent to the surfaces. The different decay rates of these charges, referred to as homo- and heterocharge, were found to be responsible for the polarity reversals observed in the early experiments.

However, it was not until Bernhard Gross made a series of fundamental experiments in the 1940's that the coexistence of the two different types of charges was convincingly demonstrated and their identity was uncovered [8,9]. His experiments not only included the isothermal measurement of polarization and depolarization currents at various temperatures and sectioning studies but he also, for the first time, applied systematically the thermally-stimulated current method to gain additional information about the two kinds of charges [8]. In these experiments he demonstrated that the heterocharge is linked to dielectric absorption involving dipoles in polar substances or ionic charges in other materials while the homocharge is due to interfacial charging of the dielectric by the adjacent electrode.

The discussion of the coexistence and interaction of dipolar and real charges has since been a central topic of electret research and has lately been of great interest in the context of the stabilization of the ferroelectric polarization of poly(vinylidene fluoride). In a more recent review, Gross summarized the early work on dielectric absorption and two-charge theory together with later work on space charge and polarization [10].

In this early work, Gross also derived some fundamental relations between the charge and polarization of electrets and the fields and currents produced. These relations were invaluable for the study of the electret charges in sectioning and thermally-stimulated current experiments, referred to above [11]. Later on, he derived from first principles the Lindmayer theorem [12] relating the current of a charged dielectric to the motion of its zero field plane [13].

\section{Irradiation effects: break- down, charge longevity, and distribution}

Throughout his scientific career, Gross had an intense interest in all aspects of radiation phenomena in dielectrics. Between his very early experiments with cosmic radiation, mentioned above, and his more recent work on charge dynamics and conductivity in irradiated dielectrics, to be discussed below, there was a period in the late 1950 's when he got interested in various phenomena caused by electron and gamma irradiation of glasses and polymers [14 - 16]. Among these were breakdown effects, charge absorption and transmission, the generation and trapping of Compton electrons, and the spatial charge distribution due to the irradiation.

One important conclusion drawn from these experiments was that the injected electrons are stored for long periods of time in the investigated dielectrics [14, 17], a notion which was initially met with considerable scepticism by some of his colleagues. Evidence of the charge retention came from breakdown phenomena observed during or after charge injection. The relevance of these findings became evident a decade or two later, when polymer films used as thermal blankets in spacecraft showed breakdown effects which led to problems in satellite communications and, in one case, to the destruction of a satellite $[18,19]$. The discharges were due to the incidence of electrons of 10 to $30 \mathrm{kV}$ energy, present in the magnetosphere in large numbers.

The generation and trapping of Compton electrons, generated by gamma rays, were topics vigorously investigated by Gross in the 1950's and 1960's. He initiated this work, coined the term Compton current [20], derived the relations between the gamma flux on one hand and the Compton current and externally measured currents on the other hand, and also studied the use of Compton currents for radiation dosimetry [21, 22]. The work of Gross stimulated numerous efforts by other laboratories, particularly on the subject of Compton diodes [23].

For more detailed studies of electrets formed by irradiation or thermal methods, Gross improved the sectioning and thermally-stimulated current (TSC) methods, which he had already used before, and combined these methods to obtain a very powerful experimental 
tool $[14,24]$. He demonstrated that by evaluating TSCs from sections of an electret, one can not only determine the spatial distribution of charge and polarization but also distinguish between the two.

Gross was thus able to show for the first time that thermally-poled carnauba wax thermo-electrets possess a uniform volume polarization [24, see also 11], while electron-irradiated borosilicate glass exhibits negative space charges due to trapped radiation electrons and positive compensation charges [14]. Even the migration of these charges, generally to the nearest electrode, could be demonstrated in these early experiments. These were probably the first quantitative studies of charge dynamics, a field which Gross pursued rigorously in his later work (see below) and which has been a topic of major, recent interest for many workers in the field.

Irradiation also releases charges in a precharged electret. Gross investigated such charge releases due to gamma irradiation and he found that the electret charge lost by heating after irradiation depends exponentially on dose [25]. These measurements, just as the ones on Compton currents discussed above, have stimulated later work on radiation dosimetry [22].

\section{Charge dynamics}

Bernhard Gross had an intense and long-lasting interest in the topic of charge motion in dielectrics. Early activities in this field were, for example, his studies of the motion of Compton electrons or of the migration of thermalized charges in irradiated dielectrics, mentioned above. In the early 1970's, Gross initiated systematic research on charge dynamics in polymers irradiated with electrons penetrating into the dielectric but not energetic enough to be fully transmitted. As before, his contributions ranged from the development of new and imaginative measuring techniques over a large variety of experimental studies to profound theoretical analyses of charge motion.

The initial focus of these studies was on phenomena occuring during charge injection, while the electron beam was still on. Gross demonstrated that the Split Faraday cup is a very powerful tool to study such charge-build-up effects [26]. In the following decades, this method was used extensively by him and by others to investigate deposition profiles, depth and drift of charge as well as radiation-induced conductivity, virtual electrode effects and other parameters of electron- and gamma-irradiated dielectrics.

Experimental results on charge dynamics were obtained by Gross and coworkers for a number of polymers, particularly for the fluorocarbon materials Teflon FEP and PTFE and for polyethylene terephthalate. He was able to show that in FEP the radiation-induced conductivity due to the primary electrons causes a significant motion of the charge centroid during and immediately after irradiation [27, 28] and that, in spite of the radiation effects, detrapping of the primary electrons plays only a minor role. If the surface of electron injection is floating, the charges move further into the dielectric in their own field, whereby their mean depth increases considerably [29].

Other experiments by Gross on charge dynamics with electron beams were concerned with carrier mobilities [30] and the transmission of primary electrons [31]. Similarly, irradiation with gamma rays and subsequent TSC measurements was employed to gain also information on carrier mobility and Schubweg [32]. In addition, Gross used controllable corona charging [33] and later a constant-current corona, for other studies of charge dynamics concerned with the injection of carriers from surface into bulk traps [34, 35].

Parallel to his experimental work on charge dynamics in the 1970's and 1980's, Gross significantly advanced the theoretical description of these phenomena. He worked on many different aspects of this field, such as the dissipation of charge layers $[36,37]$, the motion of the zero-field plane [13], the transport equation [38], space-charge limited currents [39], carrier mobilities [40], and the effect of the RIC on observed currents [41]. Of the many theoretical results achieved in these studies, only a few will be briefly discussed in the following.

These relate to the case of irradiation with an electron beam of range less than the sample thickness. Gross showed that in the irradiated region of such dielectrics the RIC contribution to the transport term by far exceeds the drift contribution [38]. He then was able to explain a puzzling observation by other workers, namely the sudden onset of currents in a biased dielectric when the electron range exceeds half the sample thickness. This so-called threshold effect was attributed to the presence of a virtual electrode at the electron range which injects carriers into the nonirradiated region and thus gives rise to a space-charge limited current [39]. Finally, he found an analytic solution to the differential equation describing electron-beam charging. 
The solution is based on the theory of linear passive relaxation systems and describes the current observed in the external circuit for different profiles of the RIC [41].

Gross' experimental and theoretical contributions to charge dynamics came at a time when this field was extremely active due to interest in radiation phenomena affecting satellite performance. His results were therefore quickly used by other workers in this field, and his counsel was eagerly sought, during this time.

\section{Radiation-Induced Conduc- tivity (RIC)}

Following his studies of charge dynamics in the 1970's, Gross became more intensely interested in various aspects of the RIC in the early 1980's. He studied electron beam as well as X-ray generated conductivities, both experimentally and theoretically and collaborated with a number of colleagues in various laboratories.

The experiments showed a complex behavior of the RIC depending on time [42-47], dose rate [43-46], electric field $[43,45,47]$, material parameters $[43,47]$, time sequences [42], etc. In particular, Gross explored the temporal dependence of the RIC and found that, in many cases, it exhibits a maximum after some time and later reaches a steady state value. While the initial rise was known to be due to an increase in the ionization of the material, he interpreted the maximum and the subsequent drop with a model assuming a transition from trap-modulated to recombination-modulated conduction [43]. He later showed that certain assumptions about the deep-trapping levels and their filling have to be made to explain all the major features of the experiments [48].

Gross' endeavor to understand radiation phenomena led him to intuitive and simple, but powerful physical models. Already in his earlier work on non-biased, electron-irradiated polymers he used a so-called box model, based on the assumption of charge deposition at the electron range, finite RIC up to the range, and zero conductivity beyond, to explain very successfully experimental data on charge buildup and charge recall [28]. When studying later the response of a biased dielectric, he used again the box model and separated the current into a radiation-driven and a voltage-driven component. He was thus able to analytically describe the currents and current reversals which he had observed in his skillful and ingenious experiments [42].
The delayed conductivity (DRIC), present after termination of the irradiation, usually persists for long periods of time. A number of measurements by Gross and coworkers $[28,43,45]$ showed that the DRIC decays as expected [49] with $(1+b t)^{-1}$, where $b$ is an inverse time constant. In other experiments Gross demonstrated that the DRIC of Teflon can be reduced by annealing such that it practically disappears and the polymer exhibits a TSC-behavior like virgin material [50]. As the frequent references to Gross' work in this field show, his contributions had a great and lasting impact on the later studies of radiation induced conduction phenomena.

\section{Electron-beam poling and secondary-electron emis- sion}

In more recent years, Bernhard Gross became interested in still other aspects of electron-beam interaction with solids. One of his imaginative ideas was to use an electron beam for the poling of piezo- and ferroelectric polymers, such as poly(vinylidene fluoride) [51]. By depositing an electron layer in the dielectric, fields are set up which will pole the material. This allows one to produce monomorphs with polarization zones of predetermined thickness and also bimorphs which can be used as bending devices [52]. Electron-beam poling was subsequently used by a number of laboratories for fundamental and applied studies and is also applied for the poling of nonlinear-optical polymers [53].

The effects of secondary emission due to electron irradiation were addressed by Gross in a whole series of papers. Already in the mid 1980's, he investigated the positive charging of polymers by irradiation with electrons of energies between the two crossover energies. In this range, the number of secondaries plus backscattered electrons exceeds the number of incoming primaries. If the sample is negatively biased to ensure that secondaries do not return to the emitting surface, a positive charging of the sample results [54]. By choosing a higher primary electron energy above the second crossover energy, Gross and coworkers were able to determine the total emission yield as a function of energy in a single charging run using constant beam energy $[55,56]$.

More recently, Gross modified this method by using a pulsed instead of a continuous electron beam [57]. 
This minimizes the accumulation of charge on the surface of the dielectric and thus makes the results for the emission yield comparable to data obtained with other methods. He was also able to show that the surface charges generated by secondary emission remain on the surface [57] and he determined the escape depth of the secondaries [58].

Very recent experimental observations to the effect that the emission yield is increased by a factor up to 10 by high electric fields [59] have been extended to the low-energy region by Gross [60]. This "stimulated" secondary emission has important implications for the radiation shielding of spacecraft. Another aspect of spacecraft shielding are thermal blankets, consisting of polymer films, which often charge up and show breakdown effects due to exposure to energetic electrons present in the magnetosphere, as mentioned above. Gross found that plasma-deposited coatings of silicon oxide or nitride on polymer films used as thermal blankets reduce breakdown effects which in turn diminishes electromagnetic interference with electronic systems on board of the spacecraft [61].

\section{Beyond electret research}

We have tried to review some of the outstanding contributions of Bernhard Gross to charge effects in solids and to show their significance for the evolution of electret research. There are, however, other aspects of Gross' work that have not yet been discussed but should at least be briefly mentioned. One is the interdisciplinarity of Gross contributions, the fact that he advanced not only electret research but in addition such diverse fields as cosmic radiation, viscoelasticity, rheology, linear system theory, and radioactive fallout. He often succeeded in applying the knowledge he had gained and the methods he had developed in one field to other fields and thus cross-fertilized all of his areas of interest. In all of his endeavors, his mastery of experiment and theory was an invaluable asset.

Another aspect of Bernhard Gross' scientific career is the intense interaction he had with many colleagues in the Americas and in Europe; over the years, he collaborated with, or worked in, many different laboratories. As a result of these collaborations, he stimulated activities in these institutions which persisted long after his assignments. This multiplication effect greatly promoted the field of electret research and helped to move it in the direction he envisioned. Thanks to his motivating personality, he inspired many generations of scientists, mostly young people, who enthusiastically carried on the work they had begun under his guidance.

Bernhard Gross, now a nonagenarian, is still following the developments in electret research with keen interest and is in frequent contact not only with the members of the Institute of Physics in So Carlos but with colleagues from around the world. We express our gratitude to him and wish him health and continued creativity for the years to come.

\section{Acknowledgements}

The author is indebted to Professors Reimund Gerhard-Multhaupt, Guilherme F. Leal Ferreira, and Roberto M. Faria for valuable comments on the manuscript.

\section{References}

1. B. Gross, Z. Phys. 107, 217 (1937).

2. B. Gross, Phys. Rev. 57, 57 (1940).

3. M. Eguchi, Proc. Phys. Math. Soc. Jap. 1, 326 (1919); Phil. Mag. 49, 178 (1925).

4. B. Gross, J. Appl. Phys. 18, 212 (1947).

5. S. Mikola, Z. Phys. 32, 475 (1925).

6. E. P. Adams, J. Franklin Inst. 204, 469 (1927).

7. A. Gemant, Philos. Mag. 20, 929 (1935).

8. B. Gross and L. F. Denard, Phys. Rev. 67, 253 (1945).

9. B. Gross, J. Chem. Phys. 17, 866 (1949).

10. B. Gross, IEEE Trans. Electr. Insul. EI-21, 249 (1986).

11. B. Gross, The sectioning technique for the determination of the volume polarization of the thermoelectret, in Static Electrification 1971 (Inst. of Physics, London), pp. $33-43$.

12. J. Lindmayer, Appl. Phys. 36, 196 (1965).

13. B. Gross and M.M. Perlman, J. Appl. Phys. 43, 853 (1972).

14. B. Gross, Phys. Rev. 107, 368 (1957).

15. B. Gross, J. Polym. Sci. 27, 135 (1958).

16. B. Gross and K. A. Wright, Phys. Rev. 114, 725 (1959).

17. B. Gross and S. V. Nablo, J. Appl. Phys. 38, 2272 (1967).

18. A. R. Frederickson, IEEE Trans. Electr. Insul. EI-18, 337 (1983).

19. P. A. Robinson and P. Coakley, IEEE Trans Electr. Insul. 27, 944 (1992).

20. B. Gross, Z. Phys. 155, 479 (1959).

21. B. Gross, IEEE Trans. Nucl. Sci. NS-25, 1048 (1978). 
22. B. Gross, Z. angew. Phys. 30, 323 (1971).

23. B. Gross, "Radiation-Induced Charge Storage and Polarization Effects", in Electrets, Topics in Applied Physics, 2nd Edition, edited by G. M. Sessler (Springer-Verlag, Heidelberg 1987), pp. 217-284.

24. B. Gross and R. J. de Moraes, J. Chem. Phys. 37, 710 (1962).

25. B. Gross and R. J. de Moraes, Phys. Rev. 126, 930 (1962).

26. B. Gross, J. Dow and S. V. Nablo, J. Appl. Phys. 44, 2459 (1973).

27. B. Gross, G. M. Sessler and J. E. West, Appl. Phys. Lett. 22, 315 (1973).

28. B. Gross, G. M. Sessler and J. E. West, J. Appl. Phys. 45, 2841 (1974).

29. B. Gross, G. M. Sessler and J. E. West, J. Appl. Phys. 48, 4303 (1977).

30. B. Gross, H. von Seggern, and R. GerhardMulthaupt, J. Phys. D: Appl. Phys. 18, 2497 (1985).

31. B. Gross, R. Gerhard-Multhaupt, K. Labonte and A. Berraissoul, Coll. \& Polym. Sci. 262, 93 (1984).

32. B. Gross, G. M. Sessler and J. E. West, J. Appl. Phys. 47, 968 (1976).

33. R. A. Moreno and B. Gross, J. Appl. Phys. 47, 3397 (1976).

34. B. Gross, J. A. Giacometti and G. F. Leal Ferreira, IEEE Trans. Nucl. Sci. NS-28, 4513 (1981).

35. B. Gross, J. A. Giacometti and G. F. Leal Ferreira, Appl. Phys. A37, 89 (1985).

36. G. F. Leal Ferreira and B. Gross, Revista Brasileira de Fisica 2, 205 (1972).

37. G.F. Leal Ferreira and B. Gross, J. Nonmetals 1, $129(1973)$.

38. B. Gross and L. N. de Oliveira, J. Appl. Phys. 45, 4724 (1974).

39. L. N. de Oliveira and B. Gross, J. Appl. Phys. 46, 3132 (1975).

40. B. Gross, Solid State Commun. 15, 1655 (1974).

41. B. Gross and G. F. Leal Ferreira, J. Appl. Phys. 50, 1506 (1979).
42. B. Gross, J. E. West, H. von Seggern and D. A. Berkley, J. Appl. Phys. 51, 4875 (1980).

43. B. Gross, R. M. Faria and G. F. Leal Ferreira, J. Appl. Phys. 52, 571 (1981).

44. A. Berraissoul, R. Gerhard-Multhaupt and B. Gross, Appl. Phys. A39, 203 (1986).

45. R. Gregorio Filho, B. Gross and R. M. Faria, IEEE Trans. Electr. Insul. EI-21, 431 (1986).

46. R.M. Faria, B. Gross and R. Gregorio Filho, J. Appl. Phys. 62, 1420 (1987).

47. R. Gregorio Filho and B. Gross, J. Appl. Phys. 66, 5478 (1989).

48. B. Gross, H. von Seggern and D.A. Berkley, Phys. Stat. Sol. (a) 79, 607 (1983).

49. J. F. Fowler, Proc. R. Soc. A236, 464 (1956).

50. B. Gross, G. M. Sessler and J. E. West, J. Appl. Phys. 46, 4674 (1975).

51. B. Gross, R. Gerhard-Multhaupt, A. Berraissoul and G. M. Sessler, J. Appl. Phys. 62, 1429 (1987).

52. G. M. Sessler and A. Berraissoul, IEEE Trans. Electr. Insul. 24, 249 (1989).

53. R. Gerhard-Multhaupt, S. Bauer, S. BauerGogonea, W. Wirges and S. Yilmaz, Proc. 8th Internat. Sympos. on Electrets, Paris (IEEE, Piscataway, 1994), pp. $775-780$

54. B. Gross, H. von Seggern and J. E. West, J. Appl. Phys. 56, 2333 (1984).

55. H. von Seggern, IEEE Trans. Nucl. Sci. NS-32, $1503(1985)$.

56. B. Gross, H. von Seggern and A. Berraissoul, IEEE Trans. Electr. Insul. EI-22, 23 (1987).

57. B. Gross and R. Hessel, IEEE Trans. Electr. Insul. 26, 18 (1991).

58. R. Hessel and B. Gross, IEEE Trans. Electr. Insul. 27, 831 (1992).

59. A. R. Frederickson and S. Woolf, J. Appl. Phys. 81, 834 (1997).

60. B. Gross, C. A. F. Pintão and R. Hessel, Brazilian J. Phys., this issue.

61. B. Gross and P. Günther, IEEE Trans. Nucl. Sci. NS-40, 83 (1993). 